\title{
OPTIMUM NONUNIFORM TRANSMULTIPLEXER DESIGN
}

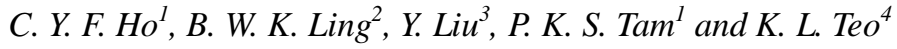

\begin{abstract}
${ }^{1}$ Department of Electronic and Information Engineering, The Hong Kong Polytechnic University, Hung Hom, Kowloon, Hong Kong, PRC. ${ }^{2}$ The Centre for Multimedia Signal Processing, Department of Electronic and Information Engineering, The Hong Kong Polytechnic University, Hung Hom, Kowloon, Hong Kong, PRC.

${ }^{3}$ Department of Applied Mathematics, The Hong Kong Polytechnic University, Hung Hom, Kowloon, Hong Kong, PRC.

${ }^{4}$ Department of Applied Mathematics and the Centre for Multimedia Signal Processing of the Department of Electronic and Information Engineering, The Hong Kong Polytechnic University, Hung Hom, Kowloon, Hong Kong, PRC.
\end{abstract}

\begin{abstract}
This paper considers an optimum nonuniform FIR transmultiplexer design subject to specifications in the frequency domain. Our objective is to minimize the sum of the ripple energy for all the individual filters, subject to the specifications on amplitude and aliasing distortions, and to the passband and stopband specifications for the individual filters. This optimum nonuniform transmultiplexer design problem can be formulated as a quadratic semi-infinite programming problem. The dual parametrization algorithm is extended to the design of this nonuniform transmultiplexer problem. If the lengths of the filters are sufficiently long and the set of decimation integers is compatible, then our algorithm guarantees that the solution obtained will give rise to the global minimum, and the required specifications are satisfied.
\end{abstract}

\section{INTRODUCTION}

Transmultiplexers are important in multimedia signal processing and communication systems because several data signals can be transmitted through a single channel by using time division multiplexing or frequency division multiplexing techniques [1]-[7]. The design of uniform transmultiplexers can be found in [1]-[5]. Since the design of uniform transmultiplexers can be based on the design of uniform filter banks, the existing theory for the design of uniform filter banks are adequate and there are many design methods available for the uniform filter banks [1]-[5]. So there are not many difficulties on the design of uniform transmultiplexers and they work very well in many applications.

Consider a specific situation, that is, when different bandwidths are allocated for different applications in the same channel. For example, a broader bandwidth is required for the transmission of videos, while a narrower bandwidth is required for transmitting texts. For another example, it may be necessary to allocate more resources to the more critical transmission applications, such as those for the military and medical purposes, while less resources are allocated to the less critical applications, such as those for the entertainments. For a further example, resources of the bandwidth can be allocated according to the rates that the consumers afford. All of these circumstances can be modeled as nonuniform transmultiplexers. The design of nonuniform transmultiplexers is actually based on the design of nonuniform filter banks [6]-[7]. However, when the set of decimation integers is incompatible, there will be only one nonzero element in at least one row of the aliasing matrix of the corresponding filter bank system. This structural dependency and the incompatibility cannot be eliminated by using a set of linear time-invariant (LTI) filters and a set of simple samplers [8]-[9]. Hence, the nonuniform transmultiplexers are suffered from amplitude, phase, aliasing and crosstalk distortions and an exact perfect reconstruction cannot be achieved [8]-[9].
Some researchers formulate the nonuniform transmultiplexer design problem as an unconstrained optimization problem such that the transmultiplexer is as close as possible to an exact perfect reconstruction system [7]. Although the unconstrained optimization problem can be solved easily, constraints are often necessary to be imposed for real practical situations. For example, as noise is always presented in the transmultiplexer, the filters with good frequency selectivity are required and the passband and stopband specifications for the individual filters are commonly imposed. Besides formulating the perfect reconstruction error as a cost function, another common way is to formulate as constraints [10]. This is because the cost function may not be convex if perfect reconstruction error is formulated as cost [11]. Moreover, amplitude and aliasing distortions may be particularly serious at certain frequency bands, instead of evenly distributed over the whole frequency spectrum [10]. Hence, by formulating the perfect reconstruction distortion as constraints, excessive amplitude and aliasing distortions at certain frequency bands can be avoided [10].

Since all the specifications are expressed as continuous constraints in the frequency domain, this optimization problem is actually a semi-infinite programming problem. To tackle a semi-infinite programming problem, one way is to approximate it as a finite dimensional problem through a discretization of the index set of the continuous constraints [12]. However, there is, in general, no guarantee that the continuous constraints will be satisfied in between these discretization points [10]. Besides, although the design problem can be converted into a semi-definite programming problem via a set of linear matrix inequalities (LMI) [13], there is no guarantee that the design procedure will converge to the global minimum [11]. Furthermore, as no constraints on the frequency selectivity of the individual filters are imposed, the frequency characteristics of the filters may become undesirable [13].

In order to avoid the above problems, we propose to solve the problem via the dual parameterization approach, which was developed recently in [10]. Based on the design of the optimum nonuniform filter bank [10], the theory and algorithm can be applied to solve this nonuniform transmultiplexer design problem. Subject to some smoothness and convexity conditions on the objective and constraint functions, the convergence of this algorithm to the global minimum is guaranteed. Moreover, this algorithm is very efficient and effective when it is applied to the design of certain types of filters. Hence, in this paper, we apply this technique to solve an optimum nonuniform transmultiplexer design problem so that the sum of the ripple energy for all the individual filters are minimized, subject to the specifications on the amplitude and aliasing distortions and to the passband and stopband specifications for the individual filters.

This paper is organized as follows. In Section 2, we give 
the notations and definitions that appear throughout this paper. In Section 3, we formulate the nonuniform transmultiplexer design problem as a quadratic semi-infinite programming problem. In Section 4, a design example is presented. Finally, we summarize and conclude our works in Section 5.

\section{NOTATIONS AND DEFINITIONS}

Let $x_{i}[n]$ and $y_{i}[n]$, for $i=0,1, \ldots, N-1$, be, respectively, the input and output signals of the nonuniform transmultiplexer as shown in Figure 1. Let $\downarrow n_{i}$ and $\uparrow n_{i}$, for $i=0,1, \ldots, N-1$, denote, respectively, the downsamplers and upsamplers, and let $H_{i}(z)$ and $F_{i}(z)$, for $i=0,1, \ldots, N-1$, be, respectively, the filters in the receiver and transmitter. The nonuniform transmultiplexer are said to be achieve perfect reconstruction if $y_{i}[n]$ is a delayed gain version of $x_{i}[n]$, for $i=0,1, \ldots, N-1$, that is, $\exists c_{i} \in \mathfrak{R}$ and $\exists m_{i} \in \mathbf{Z}$ such that $y_{i}[n]=c_{i} x_{i}\left[n-m_{i}\right]$, for $i=0,1, \ldots, N-1$, where $\mathfrak{R}$ and $Z$ denotes, respectively, the set of real numbers and integers.

\section{PROBLEM FORMULATION}

To avoid phase distortions, either symmetric or antisymmetric even-tap filters are designed, and the filters in the receiver can be expressed as:

$$
H_{i}(\omega)=2 e^{-j\left(\omega D_{i}+\phi_{i}\right)} \sum_{k=0}^{N_{i}} a_{i, N_{i}-k} \cos \left(\left(k+\frac{1}{2}\right) \omega+\phi_{i}\right),
$$

for $i=0,1, \ldots, N-1$, where $D_{i}, \phi_{i}$ and $2 N_{i}$, are respectively, the group delay, phase shift and order of the filter $H_{i}(\omega) . \phi_{i}=0$ and $\phi_{i}=\pi / 2$ correspond, respectively, to the symmetric and antisymmtric filter. Define $\mathrm{B}_{\Omega} \equiv[-\pi, \pi]$, and let the passband and stopbands of $H_{i}(\omega)$ be, respectively, denoted by $\mathrm{B}_{\mathrm{i}}^{\mathrm{p}}$ and $\mathrm{B}_{\mathrm{i}}^{\mathrm{s}}$, for $i=0,1, \ldots, N-1$. The coefficients $a_{\mathrm{i}, \mathrm{k}} \in \mathfrak{R}$ are to be designed. Let

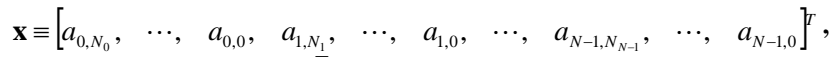
where the superscript ${ }^{\mathrm{T}}$ denotes the transpose. Define

$$
\xi_{i}(\omega) \equiv e^{-j\left(\omega D_{i}+\phi_{i}\right)} \eta_{i}(\omega),
$$

and

$$
\boldsymbol{\eta}_{i}(\omega) \equiv 2\left[\cos \left(\frac{1}{2} \omega+\phi_{i}\right), \quad \cos \left(\frac{3}{2} \omega+\phi_{i}\right), \quad \cdots, \quad \cos \left(\frac{2 N_{i}+1}{2} \omega+\phi_{i}\right)\right]^{T},
$$

for $i=0,1, \ldots, N-1$. Then,

$$
\begin{aligned}
& H_{0}(\omega)=\left[\left(\xi_{0}(\omega)\right)^{T}, \quad \mathbf{0}^{T}\right] \mathbf{x}, \\
& H_{j}(\omega)=\left[\begin{array}{lll}
\mathbf{0}^{T}, & \left(\xi_{j}(\omega)\right)^{T}, & \mathbf{0}^{T}
\end{array}\right] \mathbf{x}, \text { for } i=1,2, \ldots, N-2,
\end{aligned}
$$

and

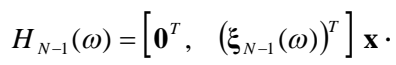

Assume the desired frequency responses are:

$$
H_{d_{i}}(\omega)=A_{d_{i}}(\omega) e^{-j \theta_{d_{i}}(\omega)} \text {, for } i=0,1, \ldots, N-1,
$$

where $A_{d_{i}}(\omega)$ and $\theta_{d_{i}}(\omega)$ are real functions representing the desired amplitude and phase responses. The cost reflecting the sum of the ripple energy for all individual filters in the passband and stopband of the filters can be expressed as:

$$
J(\mathbf{x}) \equiv \sum_{i=0}^{N-1} \int_{B_{i}^{P} \cup B_{i}^{s}}\left(\kappa_{i}(\omega)\right)^{2} d \omega
$$

where

$$
\kappa_{0}(\omega) \equiv\left[\left(\boldsymbol{\eta}_{0}(\omega)\right)^{T}, \quad \mathbf{0}^{T}\right] \mathbf{x}-A_{d_{0}}(\omega),
$$

$\kappa_{i}(\omega) \equiv\left[\begin{array}{lll}\mathbf{0}^{T}, & \left(\boldsymbol{\eta}_{i}(\omega)\right)^{T}, & \mathbf{0}^{T}\end{array}\right] \mathbf{x}-A_{d_{i}}(\omega)$, for $i=1,2, \ldots, N-2$,

and

$$
\kappa_{N-1}(\omega) \equiv\left[\mathbf{0}^{T}, \quad\left(\boldsymbol{\eta}_{N-1}(\omega)\right)^{T}\right] \mathbf{x}-A_{d_{N-1}}(\omega) \cdot
$$

It can be shown easily that there exist a positive definite matrix $\mathbf{Q}$, a vector $\mathbf{b}$ and a constant $p$ such that

$$
J(\mathbf{x})=\frac{1}{2} \mathbf{x}^{T} \mathbf{Q} \mathbf{x}+\mathbf{b}^{T} \mathbf{x}+p,
$$

where

$$
\begin{aligned}
& \mathbf{Q}=2 \operatorname{diag}\left(\mathbf{Q}_{0}^{\mathbf{c}}, \quad \mathbf{Q}_{1}^{\mathbf{c}}, \quad \cdots, \quad \mathbf{Q}_{N-1}^{\mathbf{c}}\right), \\
& \mathbf{b}=-2\left[\begin{array}{llll}
\mathbf{b}_{0}^{\mathbf{c}}, & \mathbf{b}_{1}^{\mathbf{c}}, & \cdots, & \mathbf{b}_{N-1}^{\mathbf{c}}
\end{array}\right]^{T},
\end{aligned}
$$

and

$$
p=\sum_{i=0}^{N-1} \int_{B_{i}^{P} \cup B_{i}^{s}}\left(A_{d_{i}}(\omega)\right)^{2} d \omega
$$

while

$$
\mathbf{Q}_{i}^{\mathbf{c}}=\int_{B_{i}^{P} \cup B_{i}^{s}} \boldsymbol{\eta}_{i}(\omega)\left(\boldsymbol{\eta}_{i}(\omega)\right)^{T} d \omega, \text { for } i=0,1, \ldots, N-1,
$$

and

$$
\mathbf{b}_{i}^{\mathbf{c}}=\int_{B_{i}^{p} \cup b_{i}^{B_{i}}} A_{d_{i}}(\omega)\left(\boldsymbol{\eta}_{i}(\omega)\right)^{T} d \omega \text {, for } i=0,1, \ldots, N-1 .
$$

Next, we consider the constraints to be imposed. Let the bounded on the passband and stopband ripples of $H_{i}(\omega)$ be, respectively, $\delta_{p_{i}}$ and $\delta_{s_{i}}$, for $i=0,1, \ldots, N-1$. Then the constraints on the passband and stopband ripples can be expressed as:

$$
\left|\kappa_{i}(\omega)\right| \leq \delta_{p_{i}}, \text { for } \omega \in \mathrm{B}_{\mathrm{i}}^{\mathrm{p}} \text { and for } i=0,1, \ldots, N-1,
$$

and

$$
\left|\kappa_{i}(\omega)\right| \leq \delta_{s_{i}}, \text { for } \omega \in \mathrm{B}_{\mathrm{i}}^{\mathrm{s}} \text { and for } i=0,1, \ldots, N-1 .
$$

It can be shown easily that the constraints can be expressed as follows:

$$
\mathbf{A}_{f}(\omega) \mathbf{x} \leq \mathbf{c}_{f}(\omega) \text {, for } \omega \in \mathrm{B}_{0}{ }^{\mathrm{p}},
$$

for appropriate $\mathbf{A}_{\mathbf{f}}(\omega)$ and $\mathbf{c}_{\mathbf{f}}(\omega)$.

By swapping the transmitter and receiver, we obtain a nonuniform filter bank system. It is well known that if the corresponding nonuniform filter bank system achieves perfect reconstruction with the delay of the whole system equals an integer multiple of $N$, then the corresponding transmultiplexer achieves perfect reconstruction. Hence, we can analyze the nonuniform transmultiplexer via the corresponding nonuniform filter bank system. Let

$$
F_{i}(\omega)=2 e^{-j\left(\omega D_{i}^{\prime}+\phi_{i}^{\prime}\right)} \sum_{k=0}^{N_{i}^{\prime}} b_{i, N_{i}-k} \cos \left(\left(k+\frac{1}{2}\right) \omega+\phi_{i}^{\prime}\right),
$$

for $i=0,1, \ldots, N-1$, where $D^{\prime}{ }_{i}, \phi^{\prime}{ }_{i}$ and $2 N^{\prime}{ }_{i}$, are respectively, the group delay, phase shift and order of the filter $F_{i}(\omega)$ with the coefficients $b_{\mathrm{i}, \mathrm{k}} \in \mathfrak{R}$. Define $\mathbf{y} \equiv\left[b_{0, N_{0}^{\prime}}, \cdots, \quad b_{0,0}, \quad b_{1, N_{1}^{\prime}}, \cdots, \quad b_{1,0}, \cdots, \quad b_{N-1, N_{N-1}^{\prime}}, \cdots, \quad b_{N-1,0}\right]^{T}$, then

$$
\begin{aligned}
& F_{0}(\omega)=\left[\left(\xi_{0}^{\prime}(\omega)\right)^{T}, \quad \mathbf{0}^{T}\right] \mathbf{y}, \\
& F_{j}(\omega)=\left[\begin{array}{lll}
\mathbf{0}^{T}, & \left(\xi_{j}^{\prime}(\omega)\right)^{T}, & \mathbf{0}^{T}
\end{array}\right] \mathbf{y}, \text { for } i=1,2, \ldots, N-2,
\end{aligned}
$$




$$
F_{N-1}(\omega)=\left[\mathbf{0}^{T}, \quad\left(\xi_{N-1}^{\prime}(\omega)\right)^{T}\right] \mathbf{y}
$$

where

$$
\xi_{i}^{\prime}(\omega) \equiv e^{-j\left(\omega D_{i}^{\prime}+\phi_{i}^{\prime}\right)} \eta_{i}^{\prime}(\omega)
$$

and

$$
\boldsymbol{\eta}_{i}^{\prime}(\omega) \equiv 2\left[\cos \left(\frac{1}{2} \omega+\phi_{i}^{\prime}\right), \quad \cos \left(\frac{3}{2} \omega+\phi_{i}^{\prime}\right), \quad \cdots, \quad \cos \left(\frac{2 N_{i}^{\prime}+1}{2} \omega+\phi_{i}^{\prime}\right)\right]^{T},
$$

for $i=0,1, \ldots, N-1$. Let $M$ be the least common multiple of the decimation integers. Define $k_{i}=M / n_{i}$ and

$$
\mathbf{v}_{i, j}(\omega) \equiv \frac{1}{n_{i}}\left[H_{i}\left(\omega-\frac{2 \pi j}{n_{i}}\right), \quad 0, \cdots, \quad 0\right],
$$

for $i=0,1, \ldots, N-1$, and for $j=0,1, \ldots, n_{i}-1$. There are $k_{i}-1$ zeros in the vector $\mathbf{v}_{\mathrm{i}, \mathrm{j}}(\omega)$. Let

$$
\mathbf{v}_{i}(\omega) \equiv\left[\mathbf{v}_{i, 0}(\omega), \quad \cdots, \quad \mathbf{v}_{i, n_{i}-1}(\omega)\right]^{T},
$$

for $i=0,1, \ldots, N-1$. Then, the aliasing matrix of the corresponding nonuniform filter bank system is:

$$
\mathbf{V}(\omega)=\left[\begin{array}{llll}
\mathbf{v}_{0} & (\omega), \quad \mathbf{v}_{1}(\omega), & \cdots, & \mathbf{v}_{N-1}(\omega)
\end{array}\right]
$$

Let the input of the corresponding nonuniform filter bank system be $X(\omega)$ and define

$$
\mathbf{X}_{l}(\omega) \equiv\left[X(\omega), \quad X\left(\omega-\frac{2 \pi}{M}\right), \quad \cdots, \quad X\left(\omega-\frac{2 \pi(M-1)}{M}\right)\right],
$$

then

$$
Y(\omega)=\mathbf{X}_{l}(\omega) \mathbf{V}(\omega) \mathbf{F}(\omega),
$$

where $\mathrm{Y}(\omega)$ si the output of the corresponding nonuniform filter bank system. Let

$$
\mathbf{F}(\omega) \equiv\left[F_{0}(\omega), \quad F_{1}(\omega), \quad \cdots, \quad F_{N-1}(\omega)\right]^{T} .
$$

Define

$$
\boldsymbol{\mu}(\omega) \equiv\left[\begin{array}{llll}
c e^{-j m \omega}, & 0, & \cdots, & 0
\end{array}\right]^{T},
$$

where $c$ and $m$ are, respectively, the gain and the delay of the corresponding nonuniform filter bank system. Let

$$
\mathbf{E}(\omega) \equiv|\mathbf{V}(\omega) \mathbf{F}(\omega)-\boldsymbol{\mu}(\omega)|,
$$

where $|\cdot|$ denotes the absolute values of the elements in the vector $\mathbf{V}(\omega) \mathbf{F}(\omega)-\mu(\omega)$. Define $E_{i}(\omega)$ be the $i^{\text {th }}$ element of the vector $\mathbf{E}(\omega)$. Then, the amplitude distortion of the corresponding nonuniform filter bank system is reflected by $E_{l}(\omega)$ and the aliasing distortion is reflected by $E_{i}(\omega)$ for $i=1,2, \ldots, M-1$.

Let the bounds on the amplitude and aliasing distortions be, respectively, $\delta_{a}$ and $\delta_{A}$. Then the constraints on the amplitude and aliasing distortions of the corresponding nonuniform filter bank system can be expressed as:

$$
E_{1}(\omega)=\left|\mathbf{y}^{T} \mathbf{Q}_{1}(\omega) \mathbf{x}-c e^{-j m \omega}\right| \leq \delta_{a}, \text { for } \omega \in \mathrm{B}_{\Omega},
$$

$$
E_{i}(\omega)=\left|\mathbf{y}^{T} \mathbf{Q}_{i}(\omega) \mathbf{x}\right| \leq \delta_{A}, \text { for } \omega \in \mathrm{B}_{\Omega} \text { and for } i=1,2, \ldots, M
$$

Since $\mathbf{Q}_{\mathrm{i}}(\omega)$ and $c e^{-j m \omega}$ are complex functions, we-refine the constraints as follows:

$$
\begin{aligned}
& \left|\operatorname{Re}\left\{\mathbf{y}^{T} \mathbf{Q}_{1}(\omega) \mathbf{x}-c e^{-j m \omega}\right\}\right| \leq \delta_{a}^{R}, \text { for } \omega \in \mathrm{B}_{\Omega}, \\
& \left|\operatorname{Im}\left\{\mathbf{y}^{T} \mathbf{Q}_{1}(\omega) \mathbf{x}-c e^{-j m \omega}\right\}\right| \leq \delta_{a}^{I}, \text { for } \omega \in \mathrm{B}_{\Omega},
\end{aligned}
$$

$$
\left|\operatorname{Re}\left\{\mathbf{y}^{T} \mathbf{Q}_{i}(\omega) \mathbf{x}\right\}\right| \leq \delta_{A}^{R} \text {, for } \omega \in \mathrm{B}_{\Omega} \text { and for } i=1,2, \ldots, M,
$$

and

$$
\left|\operatorname{Im}\left\{\mathbf{y}^{T} \mathbf{Q}_{i}(\omega) \mathbf{x}\right\}\right| \leq \delta_{A}^{I} \text {, for } \omega \in \mathrm{B}_{\Omega} \text { and for } i=1,2, \ldots, M,
$$

where $\operatorname{Re}\{\cdot\}$ and $\operatorname{Im}\{\cdot\}$ denote, respectively, the real and imaginary parts of the functions inside the brackets; $\delta_{a}{ }^{R}$ and $\delta_{a}{ }^{I}$ denote, respectively, the constraints on the real and imaginary parts of the amplitude distortion of the corresponding nonuniform filter bank system; $\delta_{A}{ }^{R}$ and $\delta_{A}{ }^{I}$ denote, respectively, the constraints on the real and imaginary parts of the aliasing distortion of the corresponding nonuniform filter bank system. If a set of filters in the transmitter is selected a prior, then the value of $\mathbf{y}$ are known. The constraints can be expressed as:

$$
\mathbf{A}_{r}(\omega) \mathbf{x} \leq \mathbf{c}_{r}(\omega) \text {, for } \omega \in \mathrm{B}_{0}{ }^{\mathrm{p}},
$$

for appropriate $\mathbf{A}_{\mathbf{r}}(\omega)$ and $\mathbf{c}_{\mathbf{r}}(\omega)$. Define

$$
\mathbf{A}(\omega)=\left[\begin{array}{l}
\mathbf{A}_{f}(\omega) \\
\mathbf{A}_{r}(\omega)
\end{array}\right] \text {, for } \omega \in \mathrm{B}_{0}{ }^{\mathrm{p}},
$$

and

$$
\mathbf{c}(\omega)=\left[\begin{array}{l}
\mathbf{c}_{f}(\omega) \\
\mathbf{c}_{r}(\omega)
\end{array}\right], \text { for } \omega \in \mathrm{B}_{0}{ }^{\mathrm{p}} .
$$

Consequently, the nonuniform transmultiplexer design problem can be formulated as the following semi-infinite programming problem:

$$
\min _{\mathbf{x}} \quad J(\mathbf{x})=\frac{1}{2} \mathbf{x}^{T} \mathbf{Q} \mathbf{x}+\mathbf{b}^{T} \mathbf{x}+p,
$$

subject to $\mathbf{g}(\mathbf{x}, \omega)=\mathbf{A}(\omega) \mathbf{x}-\mathbf{c}(\omega) \leq \mathbf{0}$, for $\omega \in \mathrm{B}_{0}{ }^{\mathrm{p}}$,

where $\mathbf{A}$ and $\mathbf{c}$ are continuously differentiable with respect to $\omega \in \mathrm{B}_{0}^{\mathrm{p}}$.

If the lengths of the filters are sufficiently long, then by applying the dual parameterization technique stated in [10], the solution we obtained will give the global minimum satisfying the required specifications.

\section{DESIGN EXAMPLES}

Consider a three-channel nonuniform transmultiplexer with the set of decimation integers being $\{2,4,4\}$. Let the transition bandwidths of the lowpass, bandpass and highpass filters be, respectively, $0.2751,0.2751$ and 0.1375 . Define the specifications on both the real and imaginary parts of the amplitude distortion be $-46.5028 \mathrm{~dB}$, and that of the aliasing distortion be $-77.2041 \mathrm{~dB}$. Let the specifications on the passband ripples of the lowpass, bandpass and highpass filters be, respectively, $-55.3910 \mathrm{~dB}$, $-52.7654 \mathrm{~dB}$ and $-55.9176 \mathrm{~dB}$; and that of the stopband specifications be $-35 \mathrm{~dB}$. According to our simulation, the global minimum which satisfies the required specifications is obtained. Figure 2 shows the distortions of the corresponding nonuniform filter bank system designed by both our proposed method and the method without considering the constraints.

\section{CONCLUSION}

The main contribution of this paper is to solve a nonuniform FIR transmultiplexer design problem as a semi-infinite programming problem. The method is based on the dual parameterization technique. One of the 
advantages of this method is the guaranteed convergence of the algorithm to the global minimum that satisfies the passband and stopband specifications and meets the amplitude and aliasing requirements if the solution exists. It also avoids the problems occurred in the existing semi-definite programming approaches, where the frequency responses of the filters obtained may be undesirable [13], or the continuous constraints may not be satisfied in between the discretization points [12]. The convergence property of our algorithm only requires that the orders of the filters are sufficiently large and the set of decimation integers is compatible. From our simulation results, we observe that if divergence is encountered, the indication is that the solution may not exist for the chosen set of filters. One may need to increase the orders of the individual filters or relax some other constraints and repeat the design process until the solution is found.

\section{ACKNOWLEDGEMENT}

The work obtained in this paper was supported by a research grant (project number G-YD26) from the Hong Kong Polytechnic University, and by the Centre for Multimedia Signal Processing, The Hong Kong Polytechnic University, as well as the CRGC grant (project number PolyU 5105\01E) from the Research Grants Council of Hong Kong.

\section{REFERENCES}

[1] R.D. Koilpillai, T.Q. Nguyen, and P.P. Vaidyanathan, "Some results in the theory of crosstalk-free transmultiplexers," IEEE Transactions on Signal Processing, vol. 39, no. 10, pp. 2174-2183, 1991.

[2] R.P. Ramachandran, and P. Kabal, "Bandwidth efficient transmultiplexers, part 1: synthesis," IEEE Transactions on Signal Processing, vol. 40, no. 1, pp. 70-84, 1992.

[3] R.P. Ramachandran, and P. Kabal, "Bandwidth efficient transmultiplexers, part 2: subband complements and performance aspects," IEEE Transactions on Signal Processing, vol. 40, no. 5, pp. 1108-1121, 1992.

[4] J.S. Prater, and C.M. Loeffler, "Analysis and design of periodically time-varying IIR filters, with applications to transmultiplexing," IEEE Transactions on Signal Processing, vol. 40, no. 11, pp. 2715-2725, 1992.

[5] A.N. Akansu, M.V. Tazebay, and R.A. Haddad, "A new look at digital orthogonal transmultiplexers for CDMA communications," IEEE Transactions on Signal Processing, vol. 45, no. 1, pp. 263-267, 1997.

[6] S. Coulombe, and E. Dubois, "Nonuniform perfect reconstruction filter banks over lattices with application to transmultiplexers," IEEE Transactions on Signal Processing, vol. 47, no. 4, pp. 1010-1023, 1999.

[7] T. Liu, and T. Chen, "Design of multichannel nonuniform transmultiplexers using general building blocks," IEEE Transactions on Signal Processing, vol. 49, no. 1, pp. 91-99, 2001.

[8] P.Q. Hoang, and P.P. Vaidyanathan, "Non-uniform multirate filter banks: theory and design," IEEE International Symposium on Circuits and Systems, ISCAS, vol. 1, pp. 371-374, 1989.

[9] S. Akkarakaran, and P.P. Vaidyanathan, "New results and open problems on nonuniform filter-banks," IEEE International Conference on Acoustics, Speech, and Signal Processing, ICASSP, vol. 3, pp. 1501-1504, 1999.

[10] C.Y.F. Ho, B.W.K. Ling, Y. Liu, P.K.S. Tam, and K.L. Teo, "Optimum nonuniform filter bank design via a semi-infinite programming technique," under revision in IEEE Transactions on Circuits and Systems - II: Analog and Digital Signal Processing.

[11] C.Y.F. Ho, B.W.K. Ling, Y. Liu, P.K.S. Tam, and K.L. Teo, "Optimum nonuniform FIR filter bank design by both scaling and shifting the frequency response of a prototype filter," submitted to Signal Processing.

[12] W.S. Lu, "A unified approach for the design of 2-D digital filters via semidefinite programming," IEEE Transactions on Circuits and Systems-I: Fundamental Theory and Applications, vol. 49, no. 6, pp. 814-826, 2002.

[13] A.S. Mehr, and T. Chen, "Design of nonuniform multirate filter banks by semidefinite programming," IEEE Transactions on Circuits and Systems-II: Analog and Digital Signal Processing, vol. 47, no. 11, pp. 1311-1314, 2000.
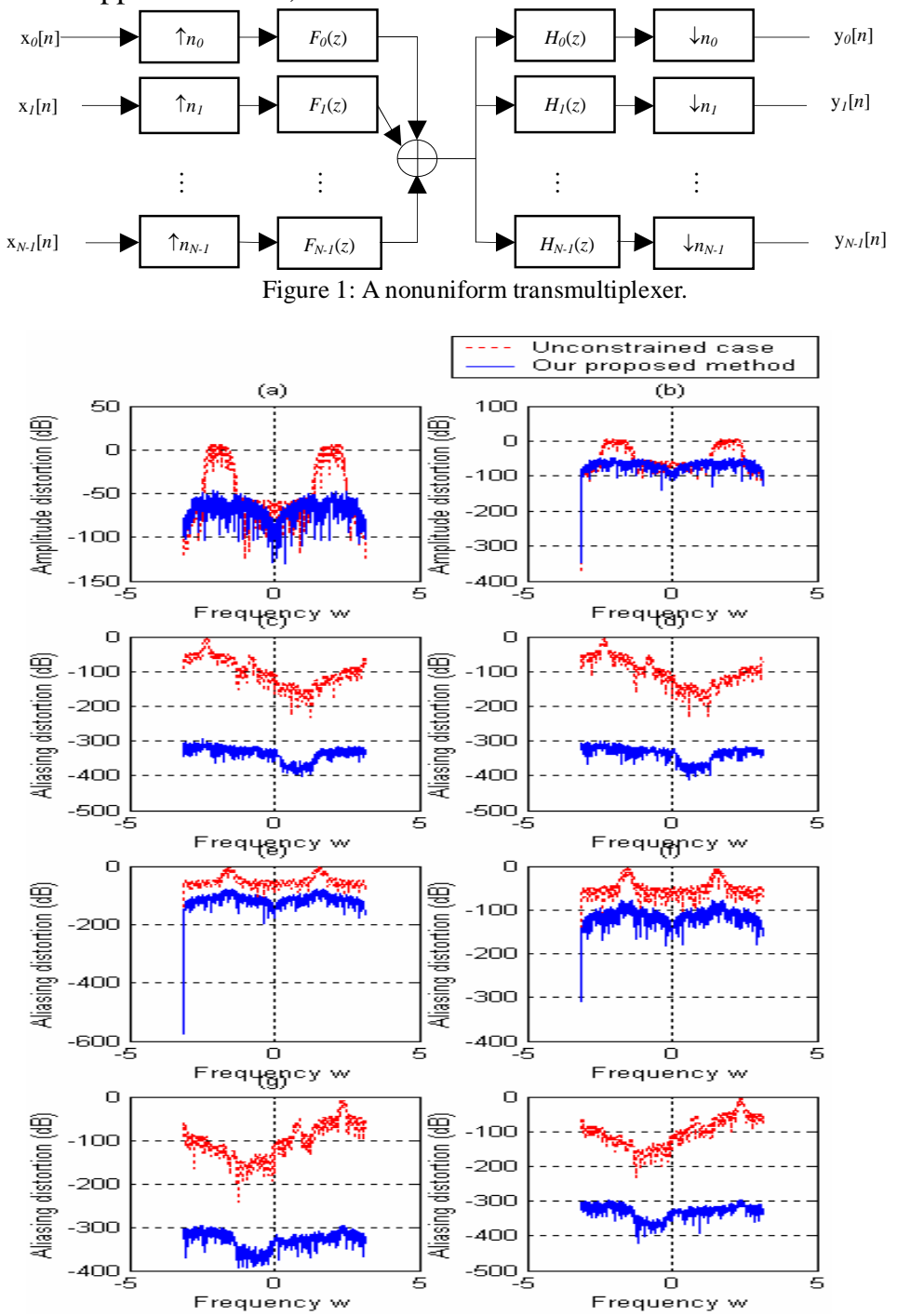

Figure 2: (a) Real part of amplitude distortion. (b) Imaginary part of amplitude distortion. (c) Real part of the first component of the aliasing distortion. (d) Imaginary part of the first component of the aliasing distortion. (e) Real part of the second component of the aliasing distortion. (f) Imaginary part of the second component of the aliasing distortion. (g) Real part of the third component of the aliasing distortion. (h) Imaginary part of the third component of the aliasing distortion. 